DRAFT VERSion August 28, 2021

Preprint typeset using $\mathrm{LT}_{\mathrm{E}} \mathrm{X}$ style emulateapj v. 11/26/04

\title{
THE EVOLUTION OF THE $M_{\mathrm{BH}}-\sigma$ RELATION INFERRED FROM THE AGE DISTRIBUTION OF LOCAL EARLY-TYPE GALAXIES AND AGN EVOLUTION
}

\author{
FRANCESCO SHANKAR ${ }^{1}$, MARIANGELA BERNARDI ${ }^{2}$, AND ZOLTÁN HAIMAN ${ }^{3}$ \\ Draft version August 28, 2021
}

\begin{abstract}
We utilize the local velocity dispersion function (VDF) of spheroids, together with their inferred agedistributions, to predict the VDF at higher redshifts $(0<z \lesssim 6)$, under the assumption that (i) most of the stars in each nearby spheroid formed in a single episode, and (ii) the velocity dispersion $\sigma$ remained nearly constant afterward. We assume further that a supermassive black hole (BH) forms concurrently with the stars, and within $\pm 1 \mathrm{Gyr}$ of the formation of the potential well of the spheroid, and that the relation between the mass of the $\mathrm{BH}$ and host velocity dispersion maintains the form $M_{\mathrm{BH}} \propto \sigma^{\beta}$ with $\beta \approx 4$, but with the normalization allowed to evolve with redshift as $\propto(1+z)^{\alpha}$. We compute the BH mass function associated with the VDF at each redshift, and compare the accumulated total $\mathrm{BH}$ mass density with that inferred from the integrated quasar luminosity function (LF; the so-called Sołtan argument). This comparison is insensitive to the assumed duty cycle or Eddington ratio of quasar activity, and we find that the match between the two $\mathrm{BH}$ mass densities favors a relatively mild redshift evolution, with $\alpha \sim 0.26$, with a positive evolution as strong as $\alpha \gtrsim 1.3$ excluded at the $99 \%$ confidence level. A direct match between the characteristic BH mass in the VDF-based and quasar LF-based BH mass functions also yields a mean Eddington ratio of $\lambda \sim 0.5-1$ that is roughly constant within $0 \lesssim z \lesssim 3$. A strong positive evolution in the $M_{\mathrm{BH}}-\sigma$ relation is still allowed by the data if galaxies increase, on average, their velocity dispersions since the moment of formation, due to dissipative processes. If we assume that the mean velocity dispersion of the host galaxies evolves as $\sigma(z)=\sigma(0) \times(1+z)^{-\gamma}$, we find a lower limit of $\gamma \gtrsim 0.23$ for $\alpha \gtrsim 1.5$. The latter estimate represents an interesting constraint for galaxy evolution models and can be tested through hydro simulations. This dissipative model, however, also implies a decreasing $\lambda$ at higher $z$, at variance with several independent studies.
\end{abstract}

Subject headings: : black hole physics - galaxies: evolution - galaxies: active

\section{INTRODUCTION}

It has now been assessed that most, if not all, local galaxies have a supermassive black hole $(\mathrm{BH})$ at their center, the mass of which is tightly correlated with the velocity dispersion $\sigma$ and other bulk properties of the host galaxy (e.g., Ferrarese \& Merritt 2000; Gebhardt et al. 2000). However, the sample of local galaxies for which the $\mathrm{BH}$ sphere of influence has been resolved amounts to only $\sim 30$. It is not clear how representative this small sample is of the whole $\mathrm{BH}$ population, and whether the correlations seen in the sample already held in the past.

Peng et al. (2006) have collected a sample of 31 lensed and 18 non-lensed Active Galactic Nuclei (AGN) at redshifts $z>1.7$. They measured rest-frame $R$-band luminosities from $H$-band fluxes and $\mathrm{BH}$ masses by applying virial relations based on emission line widths. They found that the BH-tohost galaxy luminosity at $z \sim 2$ is about the same as the one at $z \sim 0$. Therefore, once the observed rest-frame luminosity is dimmed through passive evolution to $z \sim 0$, at fixed $\mathrm{BH}$ mass the ratio $\mathrm{BH}$-to-host luminosity grows significantly, and the resulting BH-luminosity normalization is several times higher than the local one. Similar results were derived by McLure et al. (2006), who measured the BH-to-host galaxy mass ratio in a sample of radio-loud AGNs in the redshift range $0<z<2$ finding $M_{\mathrm{BH}} / M_{\mathrm{STAR}} \propto(1+z)^{2}$. Shields et al. (2006) found

\footnotetext{
${ }^{1}$ Department of Astronomy, The Ohio State University, Columbus, $\mathrm{OH}$ 43210; shankar@astronomy.ohio-state.edu

${ }^{2}$ Department of Physics and Astronomy, University of Pennsylvania, 209 South 33rd St, Philadelphia, PA 19104; bernardm@physics.upenn.edu

${ }^{3}$ Department of Astronomy, Columbia University, 550 West 120th Street, New York, NY 10027; zoltan@astro.columbia.edu
}

that the $\mathrm{CO}$ emission lines in a sample of $z>3$ quasars is very narrow, suggesting bulge mass about an order of magnitude lower than measured in the local universe, at fixed BH mass (see also Coppin et al. 2008). Treu et al. (2007) found that the $\mathrm{BH}$ masses in a sample of 20 Seyferts galaxies at $z=0.36$ are offset by an amount of $\Delta \log M_{\mathrm{BH}} \sim 0.5$ at fixed velocity dispersion, which implies an evolution of $M_{\mathrm{BH}} / M_{\mathrm{STAR}} \propto(1+z)^{1.5 \pm 1.0}$, consistent with that derived by the previous works.

On the other hand, Lauer et al. (2007) have discussed several possible biases which may seriously affect these findings. At high redshifts a sample will be biased toward the most luminous AGNs and more massive BHs. Given the observed scatter in the local relations, especially significant in the $M_{\mathrm{BH}^{-}}$ host luminosity relation, these massive BHs will be preferentially associated with the less massive, but more numerous galaxies, yielding a false sign of evolution. When the cumulative mass density of AGNs is taken into account, several authors (e.g., Haiman et al. 2004; Marconi et al. 2004; Silverman et al. 2007; Shankar et al. 2008a, hereafter SWM) have shown that once rescaled by a simple constant, it provides a good match to the cosmological star formation rate density. De Zotti et al. (2006) and SWM have shown that the galaxy stellar mass function at $z \sim 2$, mostly composed of massive early-type galaxies (e.g., Drory et al. 2005), converted into a $\mathrm{BH}$ mass density assuming a $M_{\mathrm{BH}} / M_{\mathrm{STAR}}$ ratio 3-5 times higher than in the local universe, would imply a $\mathrm{BH}$ mass density already close, if not higher, than that inferred in the local universe, leaving no room for further accretion at $z \lesssim 2$, where, in fact, a significant fraction of the total AGN energy output is produced. Recently, Ho et al. (2008) compiled a sample of 154 nearby $(z<0.1)$ active galaxies showing 
substantial ongoing $\mathrm{BH}$ growth in the most actively accreting AGNs, where BH growth appears to be delayed with respect to the assembly of the host galaxy.

In this paper, we propose a simple, yet robust, way to constrain the degree of redshift evolution in the $M_{\mathrm{BH}}-\sigma$ relation, that is relatively insensitive to assumptions that relate the SMBH population to quasars. We combine the measured velocity dispersion function (VDF) of local spheroids with a postulated power-law redshift-dependence of the $M_{\mathrm{BH}}-\sigma$ relation. By comparing the resulting total $\mathrm{BH}$ mass density at each redshift with the same quantity inferred from integration of the active galactic nuclei (AGN) luminosity function (see Sołtan 1982), we find the degree of evolution required in the $M_{\mathrm{BH}}-\sigma$ relation to match these two independent estimates. This approach yields results based on the "bulk" of the active BHs at all redshifts, and is therefore relatively insensitive to possible biases which may affect studies performed on small samples of high-redshift luminous quasars (e.g., Lauer et al. 2007). After describing the sample used in our computations in $\S 2$ we proceed to derive our main results in $\S 3$. These results are discussed further in $\S 5$, where we also offer our conclusions. Throughout this paper we use the cosmological parameters $\Omega_{m}=0.30, \Omega_{\Lambda}=0.70$, and $h \equiv H_{0} / 100 \mathrm{~km} \mathrm{~s}^{-1} \mathrm{Mpc}^{-1}=0.7$, consistent with the three(Spergel et al. 2007) and five-year (Dunkley et al. 2008) data from the Wilkinson Microwave Anisotropy Probe (WMAP).

\section{DATA}

We have used the sample of early-type galaxies obtained by Bernardi et al. (2006). The sample, extracted from the Sloan Digital Sky Survey (York et al. 2000), contains over 40,000 early-type galaxies, selected for having an apparent magnitude $14.5 \lesssim M_{r} \lesssim 17.75$, extending over a redshift range $0.013<z<0.25$, which corresponds to a maximum lookback time of 3 Gyr. The ages of galaxies are computed in two different ways, discussed in detail by Jimenez et al. (2007), from (i) single stellar population spectral fitting, using the MOPED algorithm (Heavens et al. 2000) to determine the full starformation history of the galaxies, and (ii) using the published ages by Bernardi et al. (2006) which were obtained by fitting the Thomas et al. (2005) $\alpha$-enhanced models to the Lick index absorption features measured from stacked spectra of galaxies with similar properties. The age-distributions at fixed velocity dispersion $\sigma$ are generally broad, but tend to be narrower and centered on older ages for higher values of $\sigma$. Such effects are more marked for age distributions inferred from MOPED (see Figure 1 in Haiman et al. 2007). We will compare results obtained by adopting either the MOPED or the Lick-index age distribution in $\S 3$

The analysis presented in Bernardi et al. (2006) and Haiman et al. (2007) probe velocity dispersions within $2.05 \lesssim$ $\log \left(\sigma / \mathrm{kms}^{-1}\right) \lesssim 2.45$. Here we extend such analysis including the age distributions of galaxies with velocity dispersion $2.45 \lesssim \log \left(\sigma / \mathrm{kms}^{-1}\right) \lesssim 2.55$. We find that galaxies within this last bin are even older than the oldest galaxies probed by Haiman et al. (2007), confirming and extending the general trend of increasing age for larger $\sigma$. Instead of considering one single bin with mean velocity dispersion $\log \left(\sigma / \mathrm{km} \mathrm{s}^{-1}\right)=2.5$, we have treated the bin as two distinct bins with $\log \left(\sigma / \mathrm{kms}^{-1}\right)=2.45$ and 2.55 which we have assumed share the same age distributions as the total bin. We have also included an additional bin with $\log \left(\sigma / \mathrm{kms}^{-1}\right)=$ 2.60 , which we have again assumed to have an age distribu- tion equal ${ }^{4}$ to the one with $\log \left(\sigma / \mathrm{km} \mathrm{s}^{-1}\right)=2.45$ and 2.55 (a direct estimate of the ages for these galaxies with the techniques discussed above is highly limited by the low Signalto-Noise of the spectra). As will be shown in $\S 3$ (Figure 1), this binning in $\log \sigma$ enables us to better probe the statistical evolution of the VDF even at large velocity dispersions, and it has a negligible effect in the resulting cumulative black hole mass density and on our general results.

\section{RESULTS}

We first estimate the VDF as a function of redshift $z$. At any $z$, the VDF in a given bin of velocity dispersion $\sigma_{i}$ is given by all the galaxies which have formed prior to $z$. Therefore to compute $\Phi\left(\sigma_{j}, z\right)$ we subtract from the local census of galaxies with velocity dispersion $\sigma_{i}$ those galaxies that have an age $\tau$ lower then the lookback time $\tau_{j}(z)$,

$$
\Phi\left(\sigma_{i}, z\right)=\left[1-\sum_{\tau<\tau_{j}} p\left(\tau_{j}(z) \mid \sigma_{i}\right)\right] \times \Phi\left(\sigma_{i}\right) .
$$

Note that $p\left(\tau_{j}(z) \mid \sigma_{i}\right)$ refers to the fraction of galaxies with velocity dispersion $\sigma_{i}$ which have an age of $\tau_{j}(z) \pm 1 \mathrm{Gyr}$. Therefore $\Phi\left(\sigma_{i}, z\right)$ includes in the $\sigma_{i}$ bin all galaxies whose ages are within \pm 1 Gyr of $\tau_{j}(z)$. The VDF at $z=0$ is taken from Sheth et al. (2003) and includes the contribution of bulges of spirals. We therefore assume that bulges of spirals and local spheroids within the same bin of velocity dispersion share similar age distributions. However, as discussed in $\$ 5$ below, our results would still hold even if the contribution from spirals were neglected. The statistical uncertainties associated with $\Phi(\sigma, z)$ are computed from equation (1) through error propagation including uncertainties in $p\left(\tau_{j}(z) \mid \sigma\right)$, given by Haiman et al. (2007; see their Figure 1) and $\Phi(\sigma)$, given by Sheth et al. (2003).

Figure 1 shows the VDF obtained from equation (1). The different curves in both panels show $\Phi(\sigma, z)$ at different redshifts, as labeled. At fixed redshift, the symbols indicate the position of the mean in the bin of $\log \sigma$ considered, for which reliable age distributions $p\left(\tau_{j}(z) \mid \sigma_{i}\right)$ have been computed. In the left panel of Figure 11 the $p_{j i}$ distributions have been derived from the Lick-indices method, while the right panel shows the results with the MOPED-based $p_{j i}$ distributions. In our analysis below, we will adopt the $\Phi(\sigma, z)$ implied by the $p_{j i}$ derived from Lick indices. However, we will discuss the consequences of the alternate choice on our results in $\$ 5$ In both cases, we reproduce the conclusion of previous work (e.g., Trager et al. 2000; Thomas et al. 2005; Bernardi et al. 2006; Jimenez et al. 2007; Haiman et al. 2007) - that is, we find strong evidence for downsizing: on average, galaxies with larger velocity dispersion are formed earlier. This behavior is expected from basic galaxy formation theory: highredshift galaxies form in a denser universe and therefore preferentially form out of baryonic clumps collapsed in denser, gas rich environments which in turn, induce more dissipation, more compact remnants and higher velocity dispersions. At fixed velocity dispersion, the MOPED ages are higher than inferred from Lick indices, producing a less pronounced evolution in the VDF at $0 \lesssim z \lesssim 3$. Theoretical models in which

\footnotetext{
4 a more appropriate choice would be to assign older ages to the galaxies with extreme velocity dispersions, given the general trend of older ages for higher $\sigma$, however this would pose even stronger evidence for the downsizing discussed below further strengthening our general conclusions.
} 

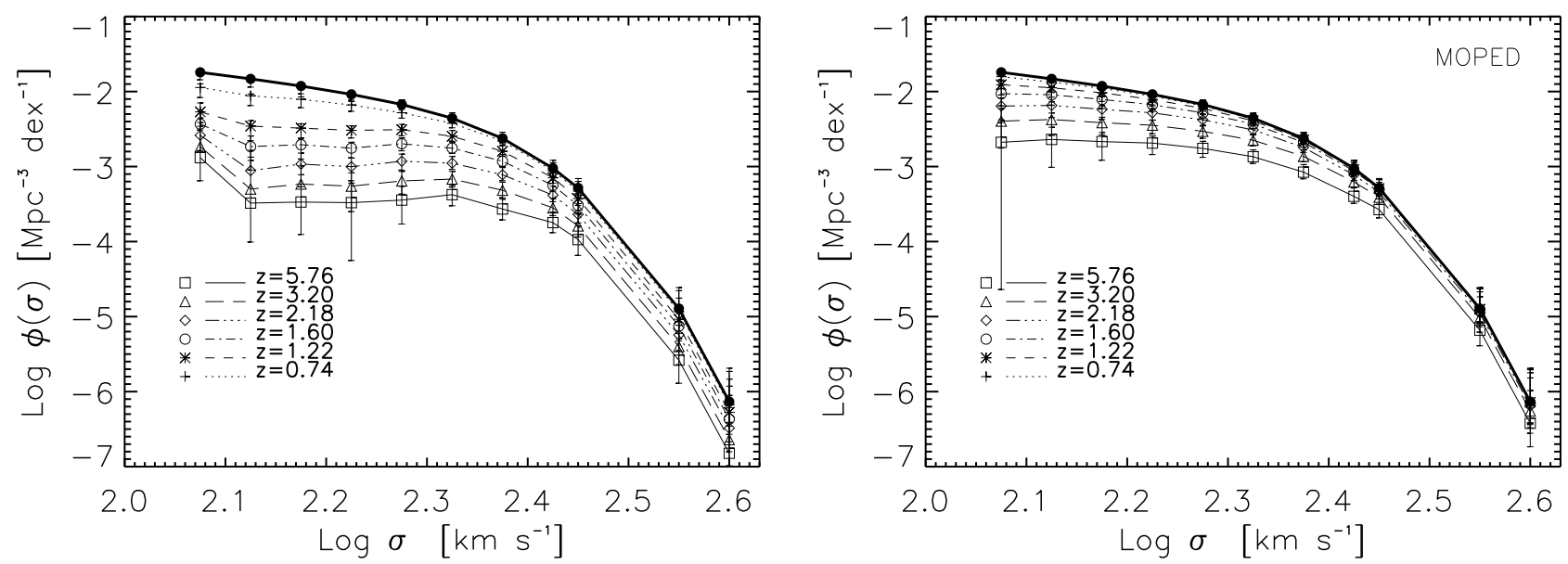

FIG. 1.- Velocity dispersion function (VDF) at different redshifts, as labeled, obtained by combining the local VDF with the age distribution of local galaxies (equation [1]). Left panel: VDF obtained using the ages computed from Lick indexes; Right panel: VDF obtained using the ages computed from the MOPED algorithm (see text for details).

the galaxy velocity dispersion is linked with the virial velocity of the host halo (e.g., Ferrarese 2002) predict similar trends for the VDF as a function of time (Cirasuolo et al. 2005; see also Loeb \& Peebles 2003).

The BH mass function implied by the VDF at any time is given by converting $\Phi(\sigma, z)$ to a $\mathrm{BH}$ mass function through the $M_{\mathrm{BH}}-\sigma$ relation and a convolution with a Gaussian with intrinsic scatter of 0.22 dex. We assume here that $\log M_{\mathrm{BH}}$ at fixed $\log \sigma$ is given by a Gaussian distribution, with a mean of

$\log \left(\frac{M_{\mathrm{BH}}}{M_{\odot}}\right)=8.21+3.83 \log \left(\frac{\sigma}{200 \mathrm{~km} \mathrm{~s}^{-1}}\right)+\alpha \log (1+z)$,

and a standard deviation of $\eta=0.22$. This latter value represents the intrinsic scatter as given by Tundo et al. (2007) and as recently confirmed by Shankar \& Ferrarese (2008). By integrating the resulting $\mathrm{BH}$ mass function at all times, we derive the total $\mathrm{BH}$ mass density $\rho_{\mathrm{VDF}}(z)$, corresponding to $\mathrm{BHs}$ in the range of $\sigma$ and $\mathrm{BH}$ mass probed by our sample at each redshift.

We then compare $\rho_{\mathrm{VDF}}(z)$ with the BH mass density obtained by direct integration of the AGN luminosity function $\Phi(L, z)$ from $z=6$ up to redshift $z$. The latter quantity is given by

$$
\rho_{\bullet}\left(>\log L_{\min }, z\right)=\frac{1-\epsilon}{\epsilon c^{2}} \int_{z}^{6} d z^{\prime} \int_{\log L_{\min }}^{\infty} \Phi\left(L, z^{\prime}\right) L \frac{d t}{d z^{\prime}} d \log L
$$

Here $\epsilon$ represents the radiative efficiency, and for our numerical calculation, we adopt the bolometric AGN luminosity function $\Phi(L, z)$ from SWM (using the LF from Hopkins et al. 2007 gives similar results as discussed below). At each redshift, we integrate equation (3) above the minimum luminosity, corresponding to the minimum $\mathrm{BH}$ mass probed by the velocity dispersion distribution in our sample via equation (2), which is $M_{\mathrm{BH}, \min } \sim 10^{7} M_{\odot}$, in the reference model. The minimum luminosity is then taken to be the Eddington (1922) luminosity corresponding to this BH mass. As we discuss in $\S 5$ below, the exact choice of the lower limit in the integral of equation (3) does not alter our conclusions.

The growth rate of an active black hole of mass $M_{\mathrm{BH}}$ is then $\dot{M}_{\mathrm{BH}}=M_{\mathrm{BH}} / t_{\mathrm{ef}}$, where the $e$-folding time is (Salpeter 1964)

$$
t_{\mathrm{ef}}=4 \times 10^{7}\left[\frac{\epsilon(1-\epsilon)^{-1}}{0.1}\right] \lambda^{-1} \mathrm{yr}
$$

where $\lambda$ is the ratio of the luminosity $\epsilon \dot{M}_{\mathrm{BH}} c^{2}$ to the Eddington luminosity. Figure 2 compares the two independent estimates of BH mass densities. The accreted mass density at each redshift obtained from $\Phi(\sigma, z)$ and the $M_{\mathrm{BH}^{-}} \sigma$ relation is shown with a solid curve. The solid squares show the redshifts where the mass density was computed. The long-dashed curve represents the mass density inferred from integration of the SWM AGN bolometric luminosity function. Given that the ages of galaxies in the sample have a median associated uncertainty of $\pm 1 \mathrm{Gyr}$, at any time $t(z)$ the BH mass density from AGNs to be compared to $\rho_{\mathrm{VDF}}(z)$ is systematically uncertain by the mass accreted within $t \pm 1 \mathrm{Gyr}$, which we show as the gray area. ${ }^{5}$ We choose a constant mean radiative efficiency of $\epsilon=0.080$, which provides a good match to the BH mass density at $z=0$ (e.g. Haiman et al. 2004, SWM). It can be immediately inferred from the left panel, which assumes an unevolving $M_{\mathrm{BH}^{-}} \sigma$ relation, that $\rho_{\mathrm{VDF}}(z)$ and $\rho_{\bullet}(z)$ are consistent with each other within errors, and therefore a strong evolution with redshift in the $M_{\mathrm{BH}^{-}} \sigma$ relation is not required. Very similar results are found if we adopt the bolometric luminosity function from Hopkins et al.(2007), shown as the dot-dashed curve in the same Figure. In this case, we use a slightly higher radiative efficiency of $\epsilon=0.104$ to renormalize the total $z=0$ accreted mass density to the local value, due to the fact that the bolometric corrections used by Hopkins et al. (2007) are about 30\% higher then those adopted by SWM. Nevertheless, even in this case we find that $\rho_{\bullet}(z)$ well matches $\rho_{\mathrm{VDF}}(z)$ at all times.

Joint confidence levels on the two parameters $\epsilon$ and $\alpha$, inferred from a $\chi^{2}$ analysis are shown in Figure 3. The cross marks the best-fit model with $\epsilon=0.08$ and $\alpha=0.26$ (corresponding to the minimum $\chi_{\min }^{2} \sim 2.6$ for 8 degrees of freedom), which is shown in the right panel of Figure 2 Once a constant radiative efficiency is fixed to match the $z=0$ local and accreted mass densities, it is evident that the available data favor a relatively mild redshift evolution of the $M_{\mathrm{BH}^{-}} \sigma$ relation with $\alpha \lesssim 0.3$, while a strong evolution with $\alpha \gtrsim 1.3$ is ruled at $99 \%$ confidence level. Likewise, negative evolution with $\alpha \lesssim-1$ is ruled out for any choice of $\epsilon$. We note

\footnotetext{
${ }^{5}$ Note that the $t \pm 1$ Gyr uncertainty is for $\rho_{\mathrm{VDF}}(z)$. However, in our calculations, assigning the uncertainty to $\rho_{\bullet}(z)$ or $\rho_{\mathrm{VDF}}(z)$ makes no difference. If the time of formation of the galaxies is uncertain by $\pm 1 \mathrm{Gyr}$, then statistically the $\rho_{\mathrm{VDF}}(z)$ at the time $t$ can be compared with the cumulative mass accreted at any time $t \pm 1 \mathrm{Gyr}$.
} 

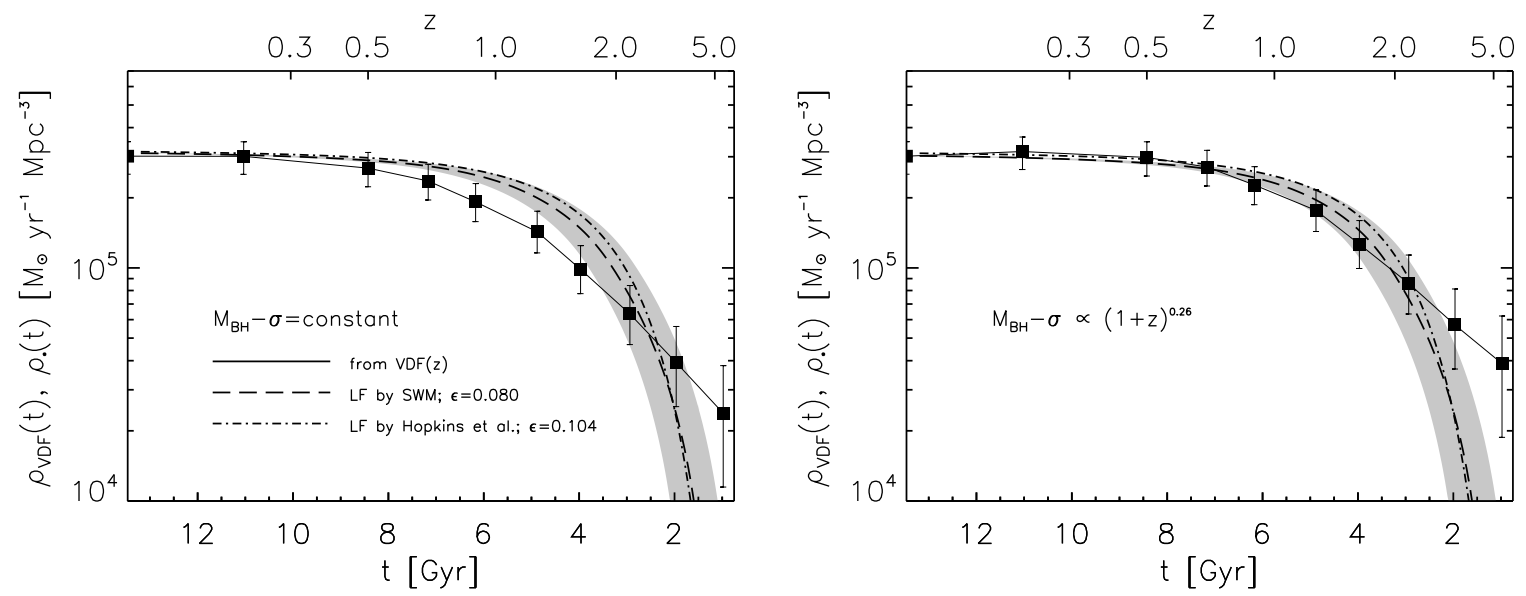

FIG. 2.-Comparison between the accreted mass density at each redshift obtained from $\Phi(\sigma, z)$, convolved with the local $M_{\mathrm{BH}}-\sigma$ relation (solid curve and solid squares) and the mass density inferred from integration of the Shankar et al. (2008a) AGN luminosity function (long dashed curve) and a radiative efficiency of $\epsilon=0.080$; the grey area represents the uncertainty at each time $t$ associated to the mass accreted within $t \pm 1$ Gyr; the dot-dashed line is the predicted accreted mass using the Hopkins et al. (2007) AGN luminosity function and a radiative efficiency of $\epsilon=0.104$. The left panel shows $\rho_{\mathrm{VDF}}(z)$ predicted from the VDF assuming that the $M_{\mathrm{BH}}-\sigma$ relation is independent of redshift, while the right panel shows the predictions for the best-fit $\rho_{\mathrm{VDF}}(z)$ when the normalization of the $M_{\mathrm{BH}}-\sigma$ relation evolves as $\propto(1+z)^{0.26}$.

that values of $\alpha \gtrsim 0.8$, in fact, yield the unphysical result that the absolute total $\mathrm{BH}$ mass density increases from $z=0$ to $z \gtrsim 0.7$, as shown in Figure 4 The confidence contours shown in Figure 3 may therefore overestimate the maximum allowed value of $\alpha$.

It is clear from equation (3) that the accreted $\mathrm{BH}$ mass density does not depend on the assumed duty cycle or Eddington ratio distribution $\lambda\left(M_{\mathrm{BH}}, z\right)$, apart from a weak dependence on the latter through the lower limit of the integration. The strongest dependencies are on the radiative efficiency and on the bolometric corrections (see also Figure 9 in SWM). On the other hand, the Eddington ratio distribution and its evolution with redshift, can be constrained by comparing the AGN-based and $\operatorname{VDF}(z)$-based differential BH mass functions (rather than comparing only the integrated quantities). The $\Phi(\sigma, z)$ convolved with the $M_{\mathrm{BH}^{-}} \sigma$ relation (equation 2) in fact, predicts the shape of the $\mathrm{BH}$ mass function for $M_{\mathrm{BH}} \gtrsim M_{\mathrm{BH}, \mathrm{min}}$. On the other hand, as extensively discussed in the literature (see SWM, and related work by, e.g. Cavaliere et al. 1982; Small \& Blandford 1992; Salucci et al. 1999; Yu $\&$ Tremaine 2002; Marconi et al. 2004; Shankar et al. 2004), if a mean Eddington ratio $\lambda=L / L_{\mathrm{Edd}}$ is assumed for the active BHs, then through a continuity equation and an assumed initial condition, the AGN luminosity function can be directly mapped into a BH mass function at all times. The "break" in the predicted $\mathrm{BH}$ mass function will then approximately reflect the break $L^{*}(z)$ in the observed AGN luminosity function, i.e., $M_{\mathrm{BH}}{ }^{*}(z) \propto L^{*} / \bar{\lambda}$, where $\bar{\lambda}$ is the mean Eddington ratio. Following Sołtan (1982) and Salucci et al. (1999), SWM (see also, e.g., Yu \& Tremaine 2002) showed that constraints on the mean radiative efficiency and Eddington ratio of BHs can be gained by comparing the directly measured and the accreted $\mathrm{BH}$ mass functions. However, the $\mathrm{BH}$ mass function has been directly measured only locally, so this comparison can be performed only at $z=0$, and can not be used to glean information on the evolution of these two parameters.

The left panel of Figure 5 compares the BH mass function predicted from the combination of $\Phi(\sigma, z)$ and the mildly evolving best-fit $M_{\mathrm{BH}}-\sigma$ relation with a normalization $\propto(1+$ $z)^{0.26}$ (shown as thick curves, with their uncertainty shown in gray), and the mass function predicted from the AGN lumi- nosity function of SWM assuming a mean $\bar{\lambda}=0.6$ (shown as thin curves). ${ }^{6}$

Figure 5 shows that up to $z \lesssim 3$, a constant (non-evolving) mean Eddington ratio of $\bar{\lambda}=0.6$ provides a good match between the shapes of the accreted $\mathrm{BH}$ mass function and the one computed from the VDF. At the low-mass end, the VDFbased BH mass function starts being incomplete, while at the high mass end, a higher intrinsic scatter in the $M_{\mathrm{BH}^{-}} \sigma$ relation and/or a more complicated Eddington ratio distribution may improve the match. Fully matching the two BH mass functions is beyond the scope of this paper (see SWM for further analysis). Our aim here is merely to demonstrate that our simple approach also provides hints on the mean Eddington ratio and its redshift evolution. Similar results are found switching to the Hopkins et al. (2007) luminosity function. The right panel of Figure 5 shows that a good match between the $\mathrm{BH}$ mass functions is recovered on adopting a constant $\lambda=1.0$. Although systematic uncertainties in the bolometric AGN luminosity function preclude tighter constraints on the mean Eddington ratio (see SWM for further discussions on these issues), it is remarkable that simple models with $0.5 \lesssim \lambda \lesssim 1.0$ constant with redshift, can provide a reasonable match with the VDF-based BH mass functions. An independent way to constrain the Eddington ratio distribution and its evolution with redshift can be derived by matching the halo clustering implied by the redshift dependent model $\mathrm{BH}$ mass function and the observed AGN clustering (Shankar et al., in preparation). We have also checked that the same values of $\lambda$ provide a good match even at $z \gtrsim 3$, however the large uncertainties associated to the VDF at these high redshifts prevent any firm conclusion.

A somewhat different version of the above exercise was performed by Haiman et al. (2007). Under the assumption that the duty cycle of quasar activity is short, Haiman et al. (2007) matched the instantaneous quasar LF at each redshift to the LF predicted from $\rho_{\mathrm{VDF}}(z)$, plus an assumed constant (non-evolving) duty-cycle and Eddington-ratio distribution. This approach neglects the $\mathrm{BH}$ mass accreted during the lu-

\footnotetext{
${ }^{6}$ Note that we assumed an initial duty cycle of 0.5 at $z=6$; however the BH mass function at $z \lesssim 3.5$ becomes independent of this assumption. See SWM for further details.
} 


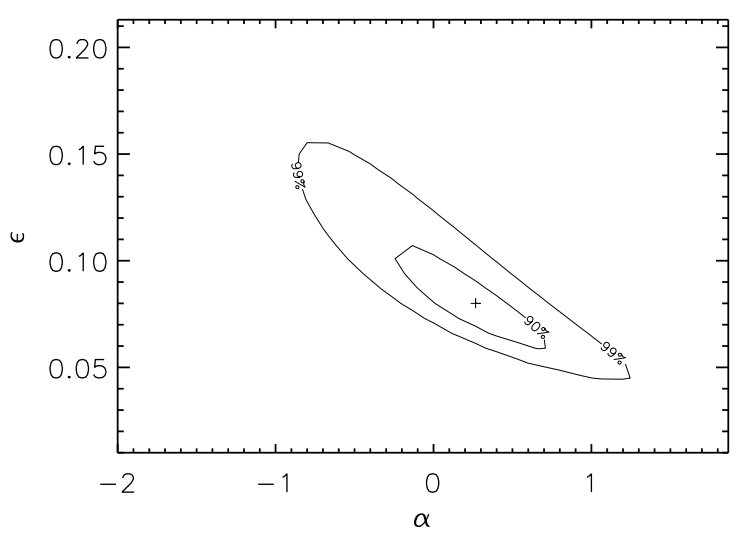

FIG. 3.- Confidence levels of $90 \%$ and $99 \%$ computed assuming $\chi^{2}=$ $\chi_{\min }^{2}+2.30$ and $\chi^{2}=\chi_{\min }^{2}+9.21$, respectively, for two parameters in the model, the radiative efficiency $\epsilon$ and the exponent $\alpha$, where the normalization of the $M_{\mathrm{BH}}-\sigma$ relation evolves as $\propto(1+z)^{\alpha}$. The cross marks the best-fit values of $\epsilon=0.08$ and $\alpha=0.26$ corresponding to the minimum $\chi_{\min }^{2} \sim 2.6$ (for 8 degrees of freedom). It is evident that a strong redshift evolution in the $M_{\mathrm{BH}}-\sigma$ relation is ruled out at a high confidence level, if the radiative efficiency is constant in time.

minous quasar phases (or at least any corresponding variation of the "quasar light-curve" caused by the growth in $\mathrm{BH}$ mass), and places a constraint directly on the relation between quasar luminosity $L$ and host velocity dispersion $\sigma$. While the $L-\sigma$ relation is essentially a convolution of the Eddington ratio distribution with the $M_{\mathrm{BH}}-\sigma$ relation, this approach cannot be used to study these two relations separately. Nevertheless, Haiman et al. (2007) found no evidence for any evolution in the $L-\sigma$ relation with redshift; their fits to the quasar LF are consistent with a constant $0.3 \lesssim \bar{\lambda} \lesssim 0.5$ combined with a nonevolving $M_{\mathrm{BH}^{-}} \sigma$ relation. Since the $M_{\mathrm{BH}^{-}} \sigma$ relation is indeed found here, independently, to be non-evolving, this breaks the degeneracy in the result of Haiman et al. (2007) and also requires that the evolution in the Eddington ratio distribution be modest.

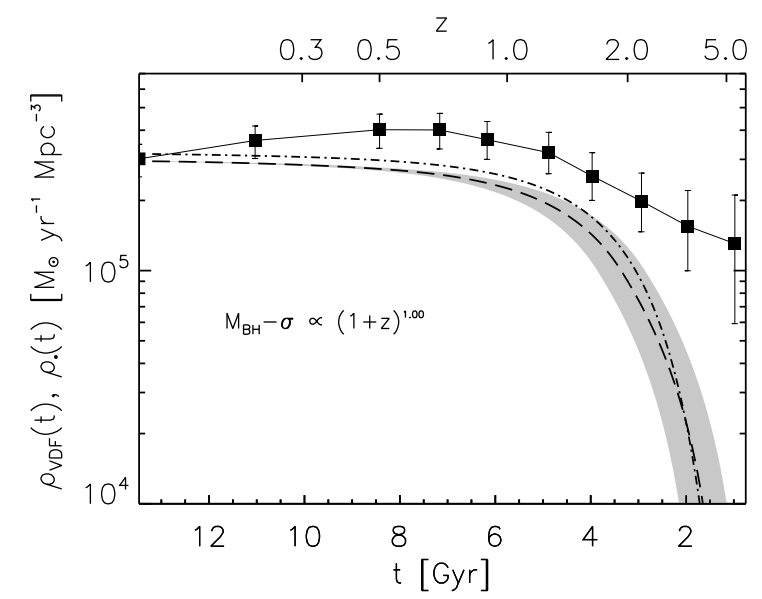

FIG. 4.- Same format as Figure 2 with parameters $\alpha=1$ and $\epsilon \sim$ $0.08,0.10$ for the SWM and Hopkins et al. (2007) luminosity functions, respectively.

\section{DISCUSSIONS}

\subsection{VARYING THE MODEL ASSUMPTIONS}

We have studied more complicated scenarios where we also allow for the scatter and/or the slope of the $M_{\mathrm{BH}}-\sigma$ relation to increase with redshift. For example, steadily increasing the slope from 3.83 to, say, 5.5, at fixed scatter of 0.22 dex, still implies $\alpha \sim 0.30$. The left panel of Figure 6 shows instead the comparison between the $\rho_{\bullet}(z)$ and the $\rho_{\mathrm{VDF}}(z)$, assuming the scatter increases with redshift from $\eta=0.22$ at $z=0$ to $\eta=0.40$ at $z=5.7$, the highest redshifts probed by our sample. It can be seen that the best-fit model requires $\alpha=0.15$, even lower than what reported in Figure 2. This is expected as these models tend to increase the $\mathrm{BH}$ mass density associated with the VDF at a given redshift, implying an even milder degree of evolution in the $M_{\mathrm{BH}}-\sigma$ normalization. The right panel of Figure 6 compares the implied mass functions predicted by the same $\eta(z)$-model and by the AGN luminosity function. Similarly to the best-fit model discussed in $\$ 3$, a good match can be recovered assuming a constant $\lambda=0.6$. Moreover, steadily increasing the intrinsic scatter from 0.22 to 0.4 dex significantly improves the match between the VDF- and AGN-based $\mathrm{BH}$ mass functions at both the high and low-mass ends.

We have also explored different models for the redshift evolution in the $M_{\mathrm{BH}^{-}} \sigma$ relation. For example, a model in which a quadratic term of the type $\delta \times \log (1+z)^{2}$ is added in equation (2) (see Wyithe 2004 for a similar test applied to the local $M_{\mathrm{BH}}-\sigma$ relation), produces a good match between $\rho_{\mathrm{VDF}}(z)$ and $\rho_{\bullet}(z)$ if $\alpha \sim \delta \sim 0.1$, with $\chi^{2} \sim 2.5$, comparable to our best-fit model. We therefore conclude that although the choice for the redshift evolution model in equation 2 is not unique, alternative solutions will still provide similar constraints on the net amount of allowed evolution.

\subsection{THE SCATTER IN THE $M_{\mathrm{BH}}-\sigma$ RELATION}

At each redshift, our approach assumes that new BHs are formed with a mass that is tightly imposed by the velocity dispersion of the host galaxy. Increasing the normalization and/or scatter of the $M_{\mathrm{BH}}-\sigma$ relation at high redshift therefore induces in the local universe a finite spread in BH mass at fixed velocity dispersion. If the evolution is steep, this can exceed the observed scatter $\eta \lesssim 0.22$ dex. Each panel in Figure 7 plots as solid lines the median $\mathrm{BH}$ mass distribution of 100 Monte Carlo simulations corresponding to a given bin of velocity dispersion, as labeled. The $\mathrm{BH}$ masses are derived from the redshift-dependent $M_{\mathrm{BH}^{-}} \sigma$ relation where the redshifts are randomly extracted from the age distribution $p_{i j}(\sigma)$ competing to each velocity dispersion. The long-dashed lines refer to the Gaussian distribution with $\eta=0.22$ measured by Tundo et al. (2007). Both our models with constant (upper panels) or evolving (lower panels) scatter still produce at $z=0$ $\mathrm{BH}$ mass distributions at fixed velocity dispersion comparable to what is observed. The small off-set in the Gaussian distributions predicted by our simulations with respect to those observed is induced by the sampling of higher redshift, more massive BHs. Models characterized by stronger redshift evolution with $\alpha \gtrsim 0.3$ will then evidently predict a scatter in the local $M_{\mathrm{BH}}-\sigma$ relation much larger than what is actually observed. Mergers are then required to be a significant component in the evolution of the BH population in these models, as the Monte Carlo simulations performed by Peng (2007) show that random $\mathrm{BH}$ mergers will tighten the relations between $\mathrm{BH}$ and host galaxy masses at late times. However, frequent mergers may, on the other hand, predict too many massive BHs with respect to those seen in the local universe (see Figure 13 in SWM). 
The main result of this paper is shown in the right panel of Figure 2, which demonstrates that a good match between $\rho_{\mathrm{VDF}}(z)$ and $\rho_{\bullet}(z)$ can be achieved by assuming a mild redshift evolution in the $M_{\mathrm{BH}^{-}} \sigma$ relation with $\alpha \lesssim 0.3$. These results are based on the age distributions $p_{j i}$ derived from Bernardi et al. (2006). However, MOPED-based galaxy ages are, on average, larger at fixed velocity dispersion, predicting a flatter dependence $\Phi(\sigma, z)$ as a function of redshift $z$ (see Figure 11. This will correspondingly flatten $\rho_{\mathrm{VDF}}(z) v s$ redshift, and decrease the best-fit $\alpha$, therefore requiring an even milder redshift evolution in the $M_{\mathrm{BH}^{-}} \sigma$ relation. A null evolution in the $M_{\mathrm{BH}}-\sigma$ relation is expected in basic AGN feedback models (e.g., Silk \& Rees 1998), in which a tight correlation derives by imposing equilibrium between the energy released by the central $\mathrm{BH}$, and the gas binding energy, linked to the velocity dispersion.

Bernardi et al. (2007), Graham (2007), and Shankar \& Ferrarese (2008) have discussed selection biases in the available sample of BHs that may induce systematic uncertainties in the determination of the local $\mathrm{BH}$ mass function. However, our conclusions are not affected by these uncertainties, because a change in the local BH mass density would be absorbed in the radiative efficiency $\epsilon$ (i.e. $\epsilon$ would be modified, to match $\rho_{\mathrm{VDF}}(z)$ and $\rho_{\bullet}(z)$ at $z=0$, but $\alpha$ would not change). By the same token, our results are only weakly dependent on whether or not the bulges of spirals are included in the estimate of the local BH mass function (a weak dependence arises only because the addition of the spiral bulges slightly skews the age-distribution of the total population to younger ages; this becomes increasingly less important toward higher redshifts, where a progressively smaller fraction of the total $\mathrm{BH}$ mass density is contributed by the low- $\sigma$ galaxies).

Likewise, uncertainties in redshift-independent bolometric corrections do not alter our conclusions. The bolometric correction adopted in SWM is lower by $\sim 30 \%$ with respect to the one used by Hopkins et al. (2007), but the sole effect of this difference is to yield a proportionally smaller value of the mean radiative efficiency to recover the match between $\rho_{\mathrm{VDF}}(z)$ and $\rho_{\bullet}(z)$ at $z=0$ (see left panel of Figure 2). Moreover, the break luminosity and bright-end slopes of the SWM and Hopkins et al. (2007) luminosity functions are somewhat different (see Figure 4 in SWM). Nevertheless, within uncertainties, the resulting $\mathrm{BH}$ accretion histories obtained from the two luminosity functions have a similar behavior with redshift, both placing the same constraint $\alpha \lesssim 0.3$ for the evolution in the normalization of the $M_{\mathrm{BH}}-\sigma$ relation.

On similar grounds, if we assume that the BHs in our sample radiate at even lower luminosities than the $L_{\min }$ considered in the integral of equation (3), our results do not change. For example, lowering the minimum luminosity to $\log L_{\min } / \mathrm{erg} \mathrm{s}^{-1}=42$, the cumulative emissivity of AGNs increases by about $\sim 30 \%$ at all redshifts yielding a very similar behavior with time. Therefore, a proportionally higher radiative efficiency plugged into equation (3) keeps the good match with the $\rho_{\mathrm{VDF}}(z)$ and specifically we find that the value of $\alpha=0.16$ yields a $\chi^{2} \sim 2.4$ for 8 degrees of freedom.

Our conclusions about the (lack of) evolution in the normalization of the $M_{\mathrm{BH}^{-}} \sigma$ relation, in general, are more dependent on redshift-dependent effects. For example, if the bolometric correction increased to high $z$ (or, e.g., if obscuration were more significant at higher redshift), this would again further decrease our favored mild positive redshift evolution in the $M_{\mathrm{BH}^{-}} \sigma$ normalization. Likewise, evolution in the mean radia- tive efficiency and/or the assumed scatter in the $M_{\mathrm{BH}}-\sigma$ relation would modify our results, in the sense that our predicted evolution would be milder if either increased toward high $z$. In principle, to allow for a stronger evolution in the $M_{\mathrm{BH}^{-}}-\sigma$ relation the radiative efficiency must significantly decrease at $z \gtrsim 3$ to boost the accreted mass density at fixed AGN luminous density. However, we have checked that $\epsilon$ must then rapidly increase at lower redshifts in order not to overproduce the local BH mass density. More quantitatively, if we set $\epsilon \sim 0.05$ at $z \gtrsim 3$, then it must be that $\epsilon \gtrsim 0.05 \times[7 /(1+z)]^{0.5}$ at lower redshifts. Such an evolution in $\epsilon$ is not enough to allow for a strong variation in the $M_{\mathrm{BH}}-\sigma$ relation. We found that $\rho_{\mathrm{VDF}}(z)$ can match the $\rho_{\bullet}(z)$ implied by the $\epsilon(z)$-model if $\alpha \sim 0.3$, which is close to our best-fit model. On other grounds, as recently shown by Shankar et al. (2008b), a too low radiative efficiency at high redshifts seems to be disfavored by $\mathrm{BH}$ accretion models which simultaneously reproduce the strong quasar clustering measured at $z=3-4$ in SDSS by Shen et al. (2007a), the mean Eddington ratio of $\lambda \gtrsim 0.5$, measured by Shen et al. (2007b) for the same quasar sample, and the high redshift quasar luminosity function (e.g., Richards et al. 2006; Fontanot et al. 2007; Shankar \& Mathur 2007).

\subsection{EVOLVING THE MAGORRIAN RELATION}

Most of the results from other groups discussed in $\S 1$ focus on the ratio between black hole mass and stellar mass. The latter may settle on longer timescales with respect to the galaxy velocity dispersion, the amplitude of which is linked to the central potential well which grows faster than the overall evolution of the halo (Zhao et al. 2003). In order to get some hints on the actual evolution of the $M_{\mathrm{BH}}-M_{\mathrm{STAR}}$ relation with redshift, we have converted the galaxy stellar mass function into a $\mathrm{BH}$ mass function assuming the $M_{\mathrm{BH}}-M_{\mathrm{STAR}}$ ratio evolving as $(1+z)^{\zeta}$. We have used the recent near-infrared stellar mass function by Pérez-González et al. (2008), well constrained within $0 \lesssim z \lesssim 3$ and $10 \lesssim \log M_{\star} / M_{\odot} \lesssim 12$. We have then converted the latter into a $\mathrm{BH}$ mass function by assuming that, on average, about $0.7 \times 10^{-3}(1+z)^{\zeta}$ (e.g., Magorrian et al. 1998; Marconi \& Hunt 2003) of the total stellar mass is locked up in spheroids and is associated to the central black hole, with a Gaussian scatter around the mean of 0.3 dex (e.g., Häring \& Rix 2004). In this case, we find that $\zeta \lesssim 0.3$ is a necessary condition for the BH mass density to be consistent with the accreted mass from AGNs, the latter derived assuming a fixed value of the radiative efficiency. This result is in agreement with the degree of evolution discussed in $\S 3$ found by evolving the $M_{\mathrm{BH}}-\sigma$ relation. Although these results are in reasonable agreement with other works (Marconi et al. 2004; Merloni et al. 2005; De Zotti et al. 2006; SWM; Merloni \& Heinz 2008), uncertainties on the lower limit of the stellar mass function and/or on the true fraction of stellar mass associated to $\mathrm{BH}$ growth at any time, make this method less reliable than the one based on velocity dispersion, and we therefore do not pursue it further.

\subsection{THE IMPACT OF MERGERS}

So far we have neglected mergers in our calculations. Major mergers between massive galaxies do occur, although recent work has suggested the galaxy merger rate may be lower than previously thought. Drory \& Alvarez (2008) compared the time variation in the stellar mass function with the evolution implied by the star formation rate alone, concluding that galaxies with stellar masses above $10^{11} M_{\odot}$ undergo at most 

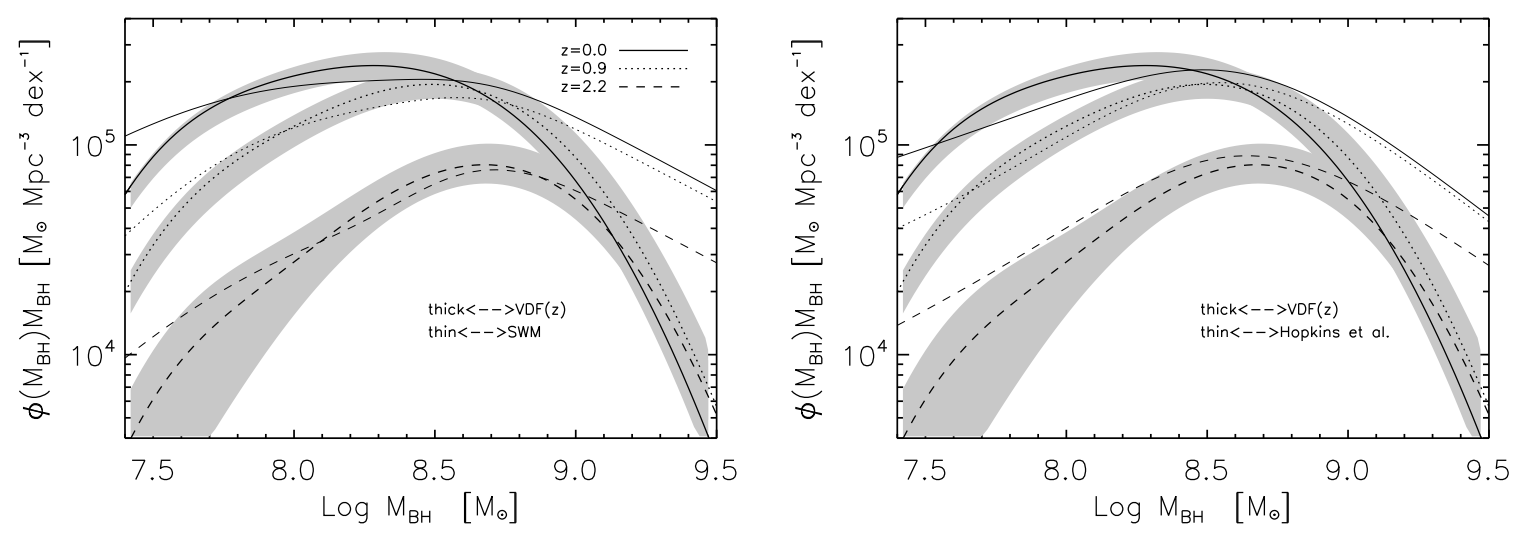

FIG. 5.- Comparison between the mass functions predicted from $\Phi(\sigma, z)$ convolved with the $M_{\mathrm{BH}}-\sigma$ relation whose normalization evolves as $\propto(1+z)^{0.26}$ (thick curves, with the uncertainty shown shaded in grey), and the mass function predicted from the AGN luminosity functions of Shankar et al. (2008; left panel) and Hopkins et al. (2007; right panel). A constant Eddington ratio of $\lambda=0.6,1.0$ has been assumed for computing the accreted mass functions in the left and right panels, respectively. It can be seen that the choice of a single constant Eddington ratio provides a good match to the velocity dispersion-based black hole mass functions, at least around the peak of the distributions. A good match is also found extending the comparison up to $z \gtrsim 3$, however the large uncertainties at these redshifts prevent any firm conclusion.

one major merger since $z \sim 1.5$, in agreement with the results of Bell et al. (2007). Lotz et al. (2006) find evidence for an even lower merger rate since $z \sim 1$ from the DEEP2 survey. Most importantly, however, a significant rate of major mergers would strengthen our conclusions. In velocity dispersion space, collisionless major mergers do not significantly affect the final $\sigma$. For example, in a dry merger of comparable-mass galaxies with mass $M_{1}$ and $M_{2}$ and corresponding velocity dispersions $\sigma_{1}$ and $\sigma_{2}$, the resulting galaxy will have a velocity dispersion $\sigma^{2} \sim\left[M_{1} \sigma_{1}^{2}+M_{2} \sigma_{2}^{2}\right] /\left(M_{1}+M_{2}\right) \lesssim \max \left(\sigma_{1}^{2}, \sigma_{2}^{2}\right)$ (e.g., Ciotti et al. 2007). Therefore, if the masses of the two galaxies are comparable, the final $\sigma$ will be close to the velocity dispersion of the progenitors.

Dry mergers would then double the number of galaxies we predict at fixed $\sigma$. Every dry major merger would in fact "split" the galaxy into two (going back in time), adding an extra galaxy with the same $\sigma$, compared to our present predictions (which neglect mergers). In turn, this implies that the merger-free $\operatorname{VDF} \Phi(\sigma, z)$ computed above for $z>0$ is a lower limit to the true abundance of galaxies with velocity dispersion $\sigma$ at redshift $z$. The associated BH mass density $\rho_{\bullet}(z)$ will consequently also be underestimated at redshift $z$. The inclusion of any mergers then predicts a larger $\mathrm{BH}$ mass density at fixed $\sigma$; to compensate for this increase, a lower normalization of the $M_{\mathrm{BH}}-\sigma$ relation at $z=1-2$ is then required, which strengthens our conclusions that large $\alpha$ values are excluded by the match between $\rho_{\mathrm{VDF}}(z)$ and $\rho_{\bullet}(z)$ (see the left panel in Figure 2).

However, if dissipation played a non-negligible role during the evolution of the galaxy (either as a result of mergers, or in isolation), then the velocity dispersion may increase with time from the epoch of first collapse. To mimic such effects, we allow all velocity dispersions to increase at higher redshifts as $\sigma(z)=\sigma(0) \times(1+z)^{-\gamma}$. Most probably this evolution is mass and/or velocity-dependent, nevertheless this approach will be able to set interesting constraints on the mean variation of $\sigma$. Also, any estimate for $\gamma$ should here be considered as a lower limit to the actual evolution of $\sigma$, as we neglect the still poorly understood increase in galaxy number density due to possible galaxy mergers. In Figure 8 we show the main results for a model with $\gamma=0.23$ and $\alpha=1.5$. Note that with the adopted scaling $M_{\mathrm{BH}} \propto \sigma^{\beta}$ with $\beta \approx 4$, we expect, a degeneracy between $\gamma$ and $\alpha$ given approximately by $\gamma \approx \alpha / \beta \approx \alpha / 4$ (although the degeneracy is modified slightly by the assumed scatter and age-spread of BHs at a given $z$ and $\sigma$ ). We find that the downsizing evolution in this case is canceled out (upper left panel), as all galaxies are now pushed to lower and lower $\sigma$ at higher redshifts.

The evolving number density in this model, shown in the upper right panel of Figure 8 , seems to be at variance with the number density evolution of early-type galaxies within $0 \lesssim z \lesssim 1$ inferred from DEEP2 by Faber et al. (2007; solid points in the same Figure). However, lacking a clear understanding of how the $\Phi(\sigma, z)$ should evolve in the presence of mergers, this model cannot be ruled out, although some inconsistencies can already be pointed out. From the Figure it can be seen that the number density of the massive red galaxies in DEEP2 at the intermediated redshifts of $0.5<z<1$, should be matched with galaxies characterized by a velocity dispersion $\sigma \lesssim 100 \mathrm{~km} \mathrm{~s}^{-1}$. Alternatively, the DEEP2 number densities could be matched with the number density of galaxies with larger velocity dispersions if mergers were a significant component in the evolution of these galaxies, thus significantly increasing their number density at higher redshifts. However, the latter hypothesis may contradict independent results (e.g., Lotz et al. 2006). The dissipative model described here also predicts a mean velocity dispersion about flat out to $z \sim 2$ and slightly decreasing at higher redshifts, as shown in the lower left panel of Figure 8 .

The main achievement of this model is the good match between $\rho_{\mathrm{VDF}}(z)$ and $\rho_{\bullet}(z)$ even if a strong evolution in the $M_{\mathrm{BH}^{-}} \sigma$ relation has been assumed $(\alpha=1.5)$, as shown in the lower right panel of Figure 8. This model is characterized by a significant dissipative phase in the evolution of typical earlytype galaxies, which could represent an interesting constraint for galaxy evolution models and it can in principle be tested through hydrodynamical simulations, which we plan to do in future work.

On the other hand, a major problem with the dissipative model is represented by its implied Eddington ratio distribution. We in fact find that the strong increase in the $M_{\mathrm{BH}^{-}} \sigma$ normalization at higher redshifts requires a significant decrease, by up to a factor of a few, in the mean Eddington ratio $\bar{\lambda}(z)$ to keep the match between the $\mathrm{BH}$ mass functions at $z \gtrsim 2$ shown in Figure 5. The latter behavior of $\bar{\lambda}(z)$ is at variance with several works which actually claim an almost constant or proba- 

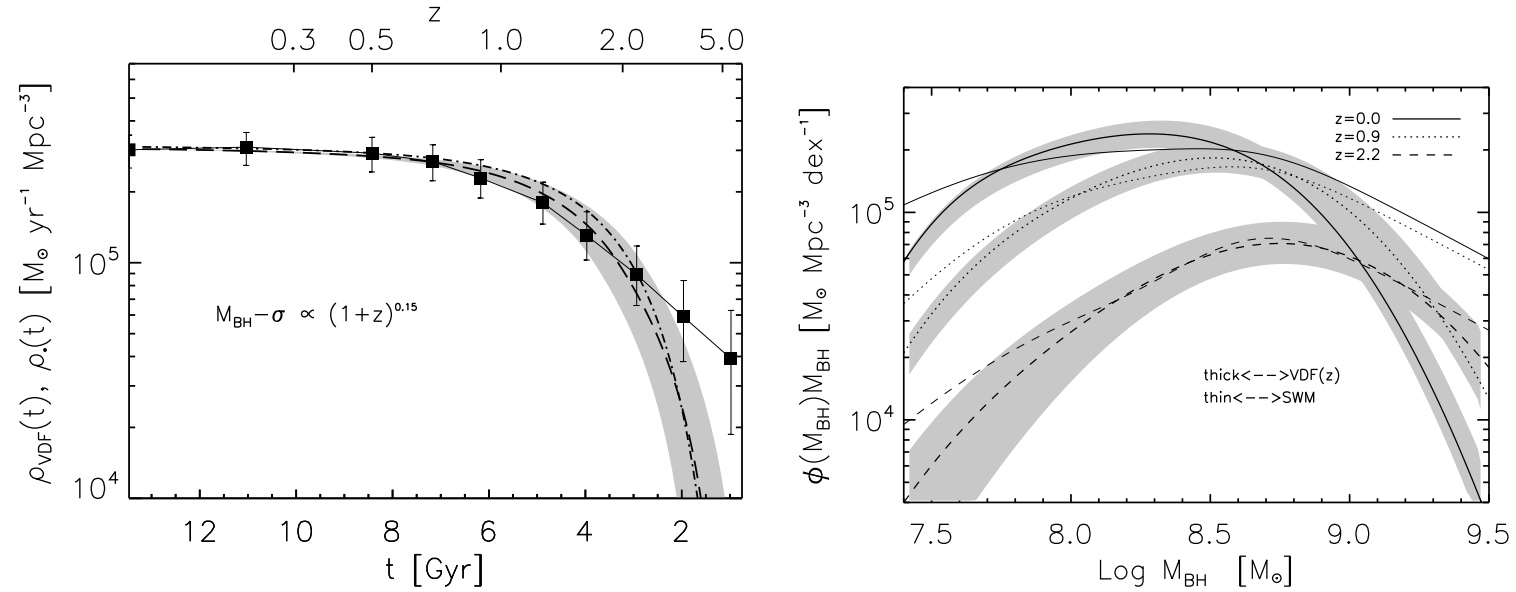

FIG. 6.- Left panel: comparison between the $\rho_{\bullet}(z)$ and the $\rho_{\mathrm{VDF}}(z)$ computed for a model in which the $\Phi(\sigma, z)$ is convolved with a $M_{\mathrm{BH}}-\sigma$ relation the scatter of which increases with redshift from $\eta=0.22$ to 0.40 . Right panel: comparison between the mass functions predicted from the same model and from the AGN luminosity functions with $\lambda=0.6$.
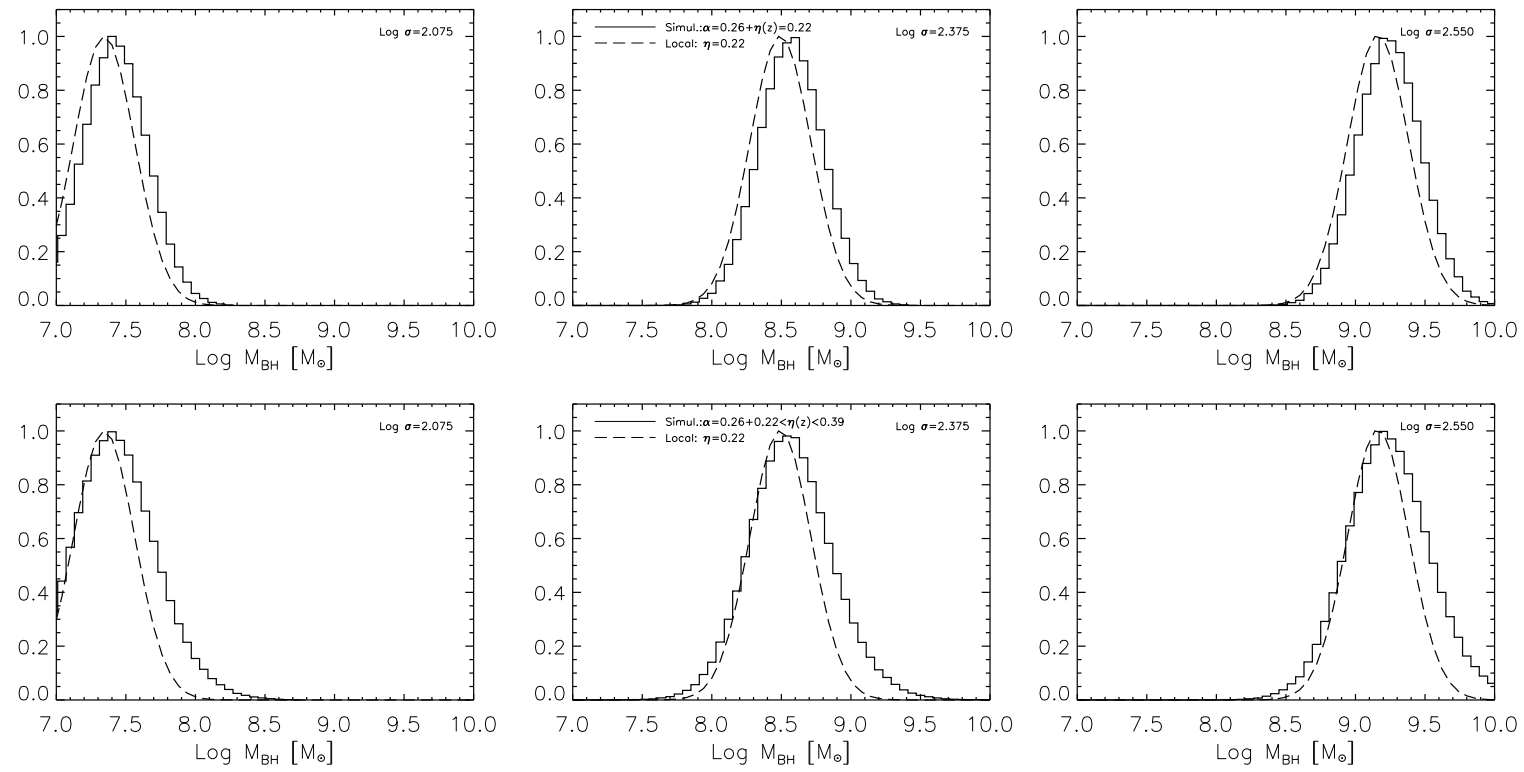

FIG. 7.- In each panel the solid histograms show the mean of a set of Monte Carlo simulations which compute from our models the expected distribution of black hole masses at fixed velocity dispersion, as labeled (see text for details). The long-dashed lines refer to the Gaussian distribution with $\eta=0.22$ measured by Tundo et al. (2007). Both our models with constant (upper panels) or evolving (lower panels) scatter still produce at $z=0$ black hole mass distributions at fixed velocity dispersion comparable to what is observed. Models characterized by stronger redshift evolution will then evidently predict a scatter in the local $M_{\mathrm{BH}^{-}} \sigma$ relation much larger than what is observed.

bly increasing $\bar{\lambda}(z)$ at higher redshifts (e.g., McLure \& Dunlop 2004; Shankar et al. 2004; Vestergaard 2004; Kollmeier et al. 2006; Netzer \& Trakhtenbrot 2007; SWM; Shen et al. 2008b; Shankar et al. 2008b).

\subsection{COMPARISON WITH PREVIOUS WORKS}

The relatively mild $M_{\mathrm{BH}}-\sigma$ redshift evolution inferred from our approach may seem in apparent disagreement with some recent independent studies. As briefly mentioned in $\S 1$, Treu et al. (2007) and Woo et al. (2008) have randomly compiled from the SDSS Data Release 4 a sample of about 20 Seyferts galaxies in the redshift range $0.37 \lesssim z \lesssim 0.57$. Their results, shown as open circles in Figure 9 are compared with those of Shen et al. (2008, shown as filled circles), who estimated the $M_{\mathrm{BH}^{-}} \sigma$ relation for a larger sample of active galaxies up to $z=0.452$. While the latter claim that no significant evolution in the $M_{\mathrm{BH}^{-}} \sigma$ relation is detectable from their sample, Woo et al. (2008) confirm the results by Treu et al. (2007) that a significant increase of $\sim 0.2$ dex in $\mathrm{BH}$ mass at fixed velocity dispersion must occur within $z=0$ and $z \sim 0.5$. Our best-fit model, shown at redshifts $z=0$ and $z=0.5$ with long-dashed and solid lines respectively, shows no strong evolution within this redshift range and it is in reasonable agreement with both samples. A significant discrepancy is noticeable with respect to the Woo et al. (2008) results for velocity dispersions log $\sigma / \mathrm{km} \mathrm{s}^{-1} \lesssim 2.3$. However, systematic uncertainties may affect these estimates; for example as also discussed by Woo et al. (2008), especially in galaxies with lower BH mass, the host galaxy contribution to the $5100 \AA$ luminosity may lead to an overestimation of the true BH mass. Overall, given the systematics and biases which affect these kind of studies (e.g., Lauer et al. 2007), we do not find strong evidence for a disagreement between these works and our results. For the same reasons, we do not attempt comparisons with the results obtained from higher redshift studies.

Merloni et al. (2004) compared the accreted BH mass den- 

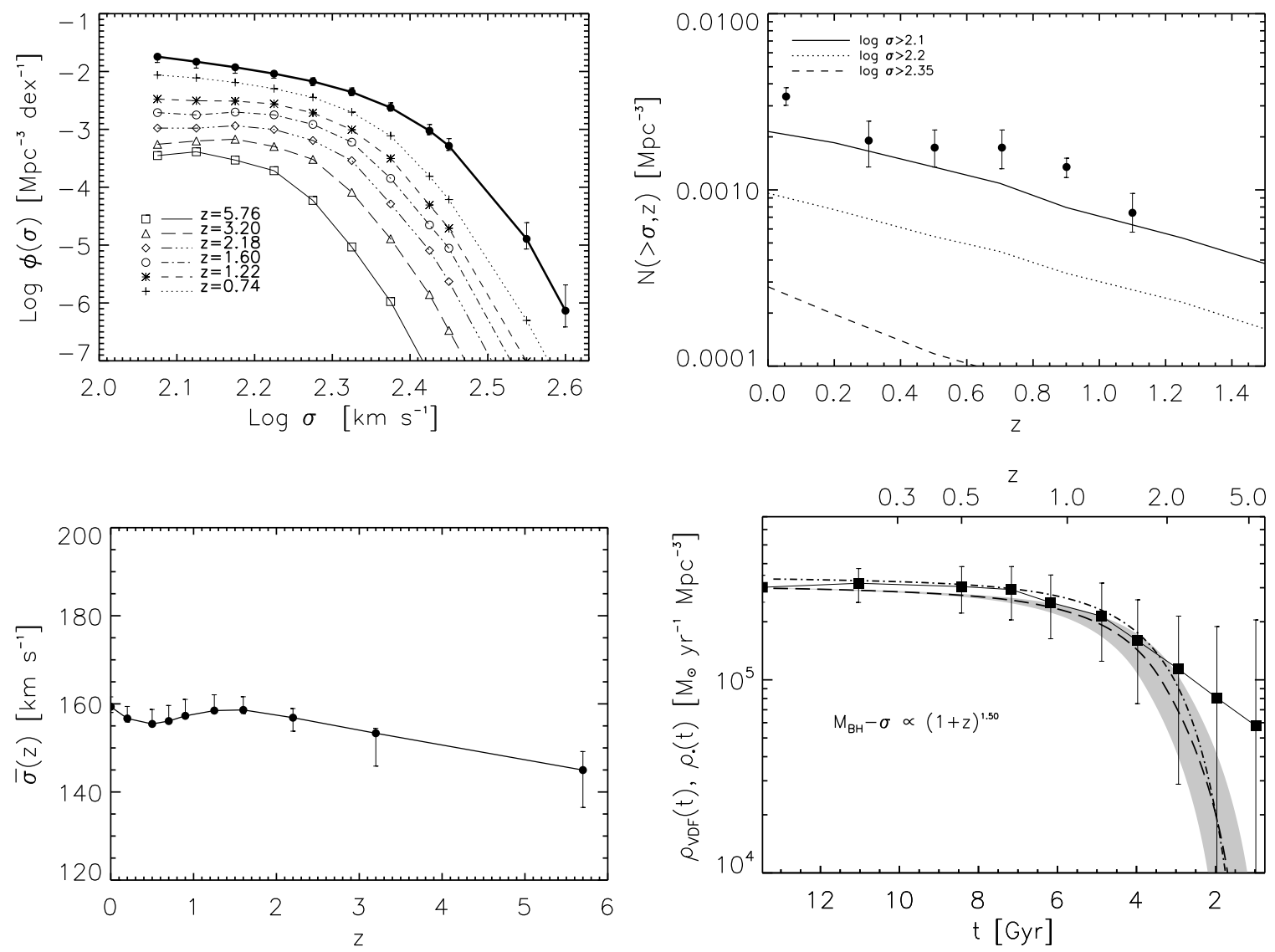

FIG. 8.- Results for a model in which we allow the mean galaxy velocity dispersion to decrease at higher redshifts to mimic the effects of prolonged wet activity in the host galaxies since their formation epoch. We assume an evolution of the type $\sigma(z)=\sigma(0) \times(1+z)^{-\gamma}$ with $\gamma=0.23$. The downsizing effect is canceled out (upper left) and the match with DEEP2 number density evolution is significantly worsen (upper right). At variance with our previous results this model predicts a mean velocity dispersion about flat out to $z \sim 2$ (lower left) and, more important, a good match between $\rho_{\mathrm{VDF}}(z)$ and $\rho_{\bullet}(z)$ with $\alpha=1.5($ lower right).

sity in AGNs with the cosmological global star formation rate density (see also Haiman et al. 2004). Although their conclusions depend on additional assumptions about the fraction of the star forming galaxies which are linked to BH growth at a given redshift, irrespective of the adopted value of the radiative efficiency their best-fit relation yields $\alpha \approx 0.5$, somewhat higher, but still consistent, with the value found here, and they also rule out $\alpha \gtrsim 1.2$ at a high confidence level. Hopkins et al. (2006) also describe a model-independent integral constraint that defines an upper limit to the allowed degree of evolution in the ratio of $\mathrm{BH}$ mass to host galaxy luminosity or mass, as a function of redshift. By comparing the AGN density with the luminosity and mass functions in different bands from redshifts $z=0-2$, they rule out at $\gtrsim 6 \sigma$ a BHhost galaxy mass ratio significantly larger at high redshifts than locally. Cattaneo \& Bernardi (2003) combined a relation between mean age and velocity dispersion, derived from a sample of SDSS local early-type galaxies, with the Sheth et al. (2003) local VDF. By assuming a redshift independent mean Eddington ratio, radiative efficiency and obscuration correction, they were then able to reproduce the AGN optical and X-ray luminosity functions. As mentioned above, similar calculations have been performed recently by Haiman et al. (2007), whose results imply, assuming a non-evolving $M_{\mathrm{BH}^{-}}$ $\sigma$ relation, that in order to reproduce the bolometric quasar luminosity function, the quasars must shine at a mean subEddington regime of $\lambda=0.5$ that is approximately constant with time. This conclusion was confirmed by the indepen- dent estimates of SWM. However, the works by Cattaneo \& Bernardi (2003) and Haiman et al. (2007) can only constrain the combination of the Eddington ratio distribution and the $M_{\mathrm{BH}}-\sigma$ relation, while our approach here can simultaneously constrain the mean accretion histories of BHs and their host galaxies, and the mean Eddington ratio of BHs at all times. A further difference is that the analysis of Haiman et al. (2007) can constrain the quasar lifetime, while the results here rely on the comparison between time-integrated quantities, and are strictly independent of the quasar lifetime.

\section{CONCLUSIONS}

In this work we combined the local VDF with the stellar age distributions estimated by Bernardi et al. (2006), to compute the VDF at higher redshifts, $\Phi(\sigma, z)$. In agreement with previous work, we find statistical evidence for downsizing, whereby the stellar populations in galaxies with higher velocity dispersions formed earlier, irrespective of the specific model we adopt for computing the galactic ages. We then computed the $\mathrm{BH}$ mass function associated with $\Phi(\sigma, z)$ at each redshift $z$, through a BH mass - velocity dispersion relation whose normalization was allowed to evolve with redshift as $\propto(1+z)^{\alpha}$. Our main underlying assumptions are that most of the growth of the central BH occurs simultaneously (within \pm 1 Gyr) with the formation of the host's potential well, and that the measured stellar ages represent this formation time to within a similar accuracy. The $\mathrm{BH}$ mass density $\rho_{\mathrm{VDF}}(z)$ inferred from the VDF can then be compared with the accu- 


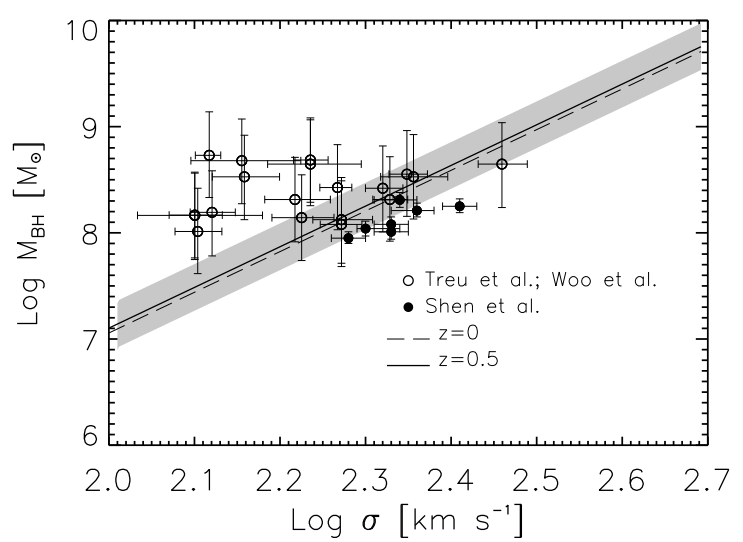

FIG. 9.- Our best-fit model for the $M_{\mathrm{BH}^{-}}-\sigma$ relation is plotted at $z=0$ and $z=0.5$, as labeled, and compared with recent data from Treu et al. (2007) and Woo et al. (2008), shown with open symbols, and Shen et al. (2008), shown with filled symbols.

mulated BH mass density implied by the time-integral of the
AGN luminosity function, $\rho_{\bullet}(z)$. We find significant evidence that the match between $\rho_{\mathrm{VDF}}(z)$ and $\rho_{\bullet}(z)$ implies a relatively mild redshift evolution, with $\alpha \lesssim 0.30$, and with values of $\alpha \gtrsim 1.3$ excluded at $99 \%$ confidence. If a positive redshift evolution stronger than $\alpha \gtrsim 1$ were to be confirmed independently in the future, then this would be a robust indication that dissipative processes played a significant role in galaxy evolution, resulting in an increase in the velocity dispersion of the spheroid components of individual galaxies with cosmic time. However, we also find evidence that a dissipative model predicts a mean Eddington ratio decreasing with increasing redshift, at variance with several independent studies.

This work was supported by NASA grants GRT000001640 (to FS), NNG04GI88G and NNX08AH35G (to ZH) and LTSA-NNG06GC19G (to MB). ZH also acknowledges support by the Polányi Program of the Hungarian National Office of Technology. FS thanks David H. Weinberg for interesting discussions.

\section{REFERENCES}

Bell, E. F., Zheng, X. Z., Papovich, C., Borch,A., Wolf, C., \& Meisenheimer, K. 2007, ApJ, 663, 834

Bernardi, M., Nichol, R. C., Sheth, R. K., Miller, C. J., \& Brinkman, J. 2006,AJ, 131, 1288

Bernardi, M., Sheth, R. K., Tundo, E., \& Hyde, J. B. 2007, ApJ, 660, 267

Cattaneo, A., \& Bernardi, M. 2003, MNRAS, 344, 45

Cavaliere, A., Giallongo, E., Vagnetti, F., \& Messina, A. 1982, A\&A, 114, 1

Ciotti, L., Lanzoni, B., \& Volonteri, M. 2007, ApJ, 658, 65

Cirasuolo, M., Shankar, F., Granato, G. L., De Zotti, G., \& Danese, L. 2005,ApJ, 629, 816

Coppin, K., et al. 2008, MNRAS, submitted, arXiv:0806.0618

De Zotti, G., Shankar, F., Lapi, A., Granato, G. L., Silva, L., Cirasuolo, M., Salucci, P., \& Danese, L. 2006, MmSAIt, 77, 661

Drory, N., Salvato, M., Gabasch, A., Bender, R., Hopp, U., Feulner, G., \& Pannella, M. 2005, ApJ, 619, 131

Dunkley, J., et al. 2008, ApJS, submitted, arXiv.org:0803.0586

Eddington, A. S. 1922, MNRAS, 83, 32

Faber, S. M., et al. 2007, ApJ, 665, 265

Fan, X., et al. 2004, ApJ, 128, 515

Ferrarese, L., \& Merritt, D. 2000, ApJ, 539, L9

Ferrarese, L. 2002, ApJ, 578, 90

Fontanot, F., Cristiani, S., Monaco, P., Nonino, M., Vanzella, E., Brandt, W. N., Grazian, A., \& Mao, J. 2007, A\&A, 461, 39

Gebhardt, K., et al. 2000, ApJL, 539, 13

Graham, A. W. 2007, MNRAS, 379, 71

Haiman, Z., Ciotti, L., \& Ostriker, J. P. 2004, ApJ, 606, 763

Haiman, Z., Jimenez, R., \& Bernardi, M. 2007, ApJ, 658, 721

Heavens, A. F., Jimenez, R., \& Lahav, O. 2000, MNRAS, 317, 965

Ho, L. C., Darling, J., \& Greene, J. E. 2008, ApJ, accepted, arXiv:0803.1952

Hopkins, P. F., Robertson, B., Krause, E., Hernquist, L., \& Cox, T. J. 2006, ApJ, 652, 107

Hopkins, P. F., Richards, G. T., Hernquist, L. 2007, ApJ, 654, 731

Jimenez, R., Bernardi, M., Haiman, Z., Panter, B., \& Heavens, A. F. 2007, ApJ, 669, 947

Kollmeier, J. A., et al. 2006, ApJ, 648, 128

Lauer, T. R., \& Tremaine, S., Richstone, D., \& Faber S. M. 2007, ApJ, 670, 249

Loeb, A., \& Peebles, P. J. E. 2003, ApJ, 589, 29

Lotz, J. M., et al. 2006, ApJ, submitted, arXiv:astroph/0602088

Marconi, A., Risaliti, G., Gilli, R., Hunt, L. K., Maiolino, R., \& Salvati, M. 2004, MNRAS, 351, 169
McLure, R. J., Dunlop., J. S. 2004, MNRAS, 352, 1390

McLure, R. J., Jarvis, M. J., Targett, T. A., Dunlop, J. S., \& Best, P. N. 2006, MNRAS, 368, 1359

Merloni, A., Rudnick, G., \& Di Matteo, T. 2005, MNRAS. 354. 37

Merloni, A., \& Heinz, S. 2008, MNRAS, accepted, arXiv:0805.2499

Netzer, H., Trakhtenbrot, B. 2007, ApJ, 654, 754

Peng, C. Y., Impey, C. D., Rix, H. W., Kochanek, C. S., Keeton, C. R., Falco,

E. E., Lehár, J., \& McLeod, B. A. 2006, ApJ, 649, 616

Richards, G. T., et al. 2006, ApJS, 166, 470

Salpeter, E. E. 1964, ApJ, 140, 796

Shankar, F., Salucci, P., Granato, G. L., De Zotti, G., \& Danese, L. 2004, MNRAS, 354, 1020

Shankar, F., \& Mathur, S. 2007, ApJ, 660, 1051

Shankar, F., Weinberg, D. H., \& Miralda-Escudé, J. 2008a, ApJ, submitted, arXiv/0710.4488 [SWM]

Shankar, F., et al. 2008b, ApJ, submitted

Shankar, F., \& Ferrarese, L. 2008, ApJ, submitted

Shen, J., Vanden Berk, D. E., Schneider, D. P., \& Hall, P. B. 2008, 135, 928

Sheth, R. K., et al. 2003, ApJ, 594, 225

Shields, G. A., Menezes, K. L., Massart, C. A., \& Vanden Bout, P. 2006, ApJ, 641,683

Silverman, J. D., et al. 2007, ApJ, submitted, arXiv:0710.2461

Small, T. A., \& Blandford, R. D. 1992, MNRAS, 259, 725

Sołtan, A. 1982, MNRAS, 200, 115

Spergel, D. N., et al. 2007, ApJS, 170, 377

Thomas, D., Maraston, C., Bender, R., \& Mendes de Oliveira, C. 2005, ApJ, 621,673

Trager, S. C., Faber, S. M., Worthey, Guy, \& González, \& J. Jesús 2000, AJ, 119,1645

Tremaine, S., et al. 2002, ApJ, 574, 740

Tundo, E., Bernardi, M., Hyde, J. B., Sheth, R. K., \& Pizzella, A. 2007, ApJ, 663,53

Vestergaard, M. 2004, ApJ, 601, 676

Woo, J. H., Treu, T., Malkan, M. A.,

Wyithe, J. S. B. 2004, MNRAS, 1082, 1098 \& Blandford, R. 2008, ApJ, in press, arXiv:0.804.0235

York, D. G., et al. 2000, AJ, 120, 1579

Yu, Q., \& Tremaine, S. 2002, MNRAS, 335, 965 